\title{
Artefact
}

Techniques, histoire et sciences humaines

2| 2014

Art et industrie : les enjeux de la formation (XVIII $\mathrm{XX}$ ' siècles) / Qu'est-ce qu'un outil simple?

\section{Les risques nucléaires sous le regard des sciences humaines et sociales}

Atomes sous surveillance. Une histoire de la sûreté nucléaire en France | Travailler dans le nucléaire. Enquête au cœur d'un site à risques

\section{Soraya Boudia}

\section{(2) OpenEdition}

\section{Journals}

\section{Édition électronique}

URL : https://journals.openedition.org/artefact/8658

DOI : 10.4000/artefact.8658

ISSN : 2606-9245

Éditeur :

Association Artefact. Techniques histoire et sciences humaines, Presses universitaires du Midi

Édition imprimée

Date de publication : 11 septembre 2014

Pagination : 195-201

ISBN : 978-2-271-08150-6

ISSN : 2273-0753

\section{Référence électronique}

Soraya Boudia, « Les risques nucléaires sous le regard des sciences humaines et sociales », Artefact [En ligne], 2 | 2014, mis en ligne le 11 mai 2021, consulté le 24 août 2021. URL : http:// journals.openedition.org/artefact/8658 ; DOI : https://doi.org/10.4000/artefact.8658

Ce document a été généré automatiquement le 24 août 2021.

\section{(c) (i) $(9)$}

Artefact, Techniques, histoire et sciences humaines est mise à disposition selon les termes de la Licence Creative Commons Attribution - Pas d'Utilisation Commerciale - Pas de Modification 4.0 International. 


\section{Les risques nucléaires sous le regard des sciences humaines et sociales}

Atomes sous surveillance. Une histoire de la sûreté nucléaire en France | Travailler dans le nucléaire. Enquête au cœur d'un site à risques

\section{Soraya Boudia}

\section{RÉFÉRENCE}

Cyrille Foasso, Atomes sous surveillance. Une histoire de la sûreté nucléaire en France, Bruxelles, Bern, Berlin, Frankfurt am Main, New York, Oxford, Wien, 2012, 542 p. Pierre Fournier, Travailler dans le nucléaire. Enquête au cœur d'un site à risques, Paris, Armand Colin, 2012, 232 p.

1 L'accident de Fukushima a de nouveau braqué les projecteurs sur l'industrie nucléaire et les risques qu'elle peut engendrer. Après de nombreux débats publics, des témoignages alarmants, des critiques militantes renouvelées et des productions culturelles et médiatiques, les sciences humaines sociales investissent à nouveau la thématique du nucléaire, en particulier les questions de risques. Ces derniers sont aujourd'hui saisis par un ensemble de travaux mobilisant aussi bien des historiens, des sociologues, des anthropologues, des politistes, des juristes ou des économistes et donnant lieu à un ensemble de publications portant aussi bien sur l'analyse de l'accident de Fukushima et ses répercutions sur les modes d'existence industrielle et politique du nucléaire que sur les trajectoires historiques du nucléaire, ses transformations et ses permanences. Les deux ouvrages présentés ici ont été publiés après l'accident de Fukushima, mais ils sont le résultat de travaux engagés de longue date par des chercheurs ayant une excellente connaissance du terrain du nucléaire. Ils ont d'emblée le mérite, par rapport aux nombreuses publications sur le nucléaire, de ne pas s'arrêter uniquement à l'étude des discours publics, mais d'investir effectivement leur terrain, à partir de l'exploration de fonds d'archives, la réalisation d'observations ethnographiques ou la conduite d'entretiens. Ils offrent de ce fait des éclairages 
saisissants de ce que signifient les risques nucléaires dans leur variété pour les différents acteurs et institutions œuvrant dans ou autour du nucléaire.

2 Le premier ouvrage, Atomes sous surveillance, est issu d'une thèse de doctorat soutenue en 2003. Son projet est ambitieux : retracer l'histoire longue des concepts de la sûreté et ses modes d'organisation institutionnelle en France. L'auteur débute son récit en 1939, après la découverte de la fission nucléaire et l'achève avec l'accident de Fukushima couvrant ainsi soixante-dix ans d'une histoire riche en réalisations techniques, en accidents mineurs et majeurs, en contestations publiques et configurations et reconfigurations institutionnelles de l'expertise et de la régulation. Il nous fait pénétrer dans l'univers complexe de scientifiques et d'ingénieurs qui ont élaboré les principes et les techniques censés maitriser les risques des centrales nucléaires. Il nous propose ainsi une « histoire technique des techniques » qui analyse et décortique les différents concepts formalisés dans ce qui a été nommé «sûreté nucléaire ».

L'ouvrage offre un panorama de la naissance de la sûreté, d'abord aux États-Unis et en Grande-Bretagne, puis en France. Il insiste sur la démarche empiriste qui caractérise les approches de la sûreté jusqu'à la fin des années 1950. Dans le cas de la France, il montre que la question de la sûreté des réacteurs a été une préoccupation portée par quelques individus dès les débuts du Commissariat à l'énergie nucléaire (CEA), fondé en 1945. Toutefois, c'est surtout à partir du milieu des années 1950 que cette problématique fait l'objet d'une professionnalisation et d'une institutionnalisation à la faveur du développement d'une expertise et d'une réglementation internationale sous l'égide de deux organisations, Euratom en Europe et l'Agence internationale pour l'énergie nucléaire (AIEA) à l'échelle internationale. L'accident de Winscale en Grande-Bretagne vient appuyer les premières formalisations des démarches de sûreté. Ceci se traduit en France par la création de la Commission de sûreté des installations atomiques (CSIA) en 1960. Celle-ci a pour mission l'examen de la sûreté des installations existantes et à venir. La profession se structure alors avec une expertise endogène au CEA, une conception de l'expertise en sûreté défendue par de nombreux scientifiques et gestionnaires du nucléaire. Elle s'appuie sur l'idée qu'un expert doit être présent au sein même des équipes en charge de la conception et de la mise en œuvre des réacteurs pour contribuer à l'ensemble du processus et l'accompagner. Cette période est surtout marquée par des améliorations techniques, au premier rang desquelles figurent l'automatisation de certaines opérations et l'installation de capteurs. Cette période est également marquée par la formalisation des procédures avec, en particulier, la rédaction d'un " rapport de sûreté ", consignant l'ensemble des mesures envisagées en réponse aux différents scénarios d'accidents. Cette expertise est très souvent partagée par les différents pays impliqués dans un programme nucléaire, ce qui débouche sur des visions convergentes et l'adoption d'un ensemble de concepts communs, comme le concept de « défense en profondeur » ou celui des «barrières » et leur tenue successive en cas d'accident dans le cœur du réacteur.

4 L'ouvrage analyse ensuite de manière intéressante les transformations de l'expertise en sûreté nucléaire à la suite du développement industriel du programme électronucléaire. Le passage d'une phase de conception scientifique et technique à la construction effective d'un ensemble de centrales nucléaires pour la production de l'électricité voit l'arrivée d'acteurs industriels, en premier lieu l'entreprise Électricité de France (EDF). Ces transformations donnent lieu à des luttes entre le CEA et EDF, 
notamment sur les modalités d'analyse et d'inspection dans le domaine de la sûreté. Le CEA, détenant l'expertise nécessaire, souhaite exercer un contrôle de la sûreté sur l'ensemble du programme électronucléaire français. EDF sort victorieuse de cet épisode avec une nouvelle formalisation des processus d'expertise et la création au sein du ministère de l'Industrie, et non du CEA, du Service central de sûreté des installations nucléaires (SCSIN), en 1973. Toutefois, ce service ne débute ses activités qu'avec trois ingénieurs, les capacités d'expertise technique restant concentrées au CEA. En cela, la situation française tranche avec les recompositions institutionnelles aux États-Unis où la nouvelle commission de régulation du nucléaire est dotée d'une expertise propre, issue de l'institution homologue du CEA, l'Atomic Energy Commission. Le processus de reconfigurations en France débouche néanmoins sur une division du travail qui dessine une « organisation à la française » de l'expertise et du contrôle de la sûreté nucléaire. Tout d'abord un Département de sûreté nucléaire est créé au sein du CEA, puis une nouvelle étape est franchie en 1976 avec la création de l'Institut de protection et de sûreté nucléaire qui regroupe les experts de sûreté et de radioprotection du CEA. L'IPSN est alors en charge de l'expertise tandis que le SCIN au ministère de l'Industrie a la responsabilité de la prise de décision relative aux questions de sûreté.

5 Ces différentes transformations institutionnelles prennent place dans une période de contestations qui voit l'essor d'un mouvement anti-nucléaire international. Les experts et les administrations en charge des risques nucléaires tendent à minimiser le rôle de cette critique dans les reconfigurations que connait l'ensemble du secteur nucléaire, les présentant comme des évolutions endogènes dictées par la logique de développement du secteur. Sans reprendre à son compte cette analyse, l'auteur montre l'importance des changements techniques au cours de cette période avec, en particulier, le transfert de technologies américaines vers la France. Il montre également l'importance des révisions et des extensions de la doctrine et des concepts de sûreté à partir de la fin des années 1970. Il examine les sources de ces transformations: d'une part, des grands accidents que commencent à connaître le secteur et, d'autre part, des retours d'expériences des difficultés d'exploitation du parc nucléaire. Le premier accident qui joue un rôle déterminant dans la révision de la doctrine de la sûreté est celui de Three Mile Island, aux États-Unis, en 1979. Cet événement fait l'effet d'un choc, rendant palpable la possibilité de fusion du cœur d'un réacteur. Il débouche sur une remise en cause de l'approche de la sûreté avec une focalisation plus importante sur le rôle d'agents dans l'enclenchement d'accidents graves. Le second accident majeur est celui de Tchernobyl, en 1986. Si les experts occidentaux insistent sur l'obsolescence des technologies soviétiques et le laxisme des autorités, la possibilité d'un accident grave s'installe désormais dans le paysage de la sûreté. Ces différents accidents, ainsi que les difficultés techniques du parc électronucléaire français - devenues visibles par la multiplication des sources d'expertise ainsi que le renouvellement de critiques multiformes du nucléaire (conditions de travail, manque d'information, non transparence...) - complexifient le monde dans lequel évoluent désormais les acteurs de la sûreté. L'ouvrage s'achève en dressant un nouveau panorama des transformations de l'expertise en sûreté nucléaire, ancré dans une nouvelle vague de reconfigurations institutionnelles en France au début des années 2000. Ces dernières sont marquées par une séparation plus importante entre les producteurs (CEA, EDF) et les activités d'expertise et de contrôle exercées désormais par le nouvel Institut de radioprotection et de sûreté (IRSN). L'IRSN regroupe désormais l'ensemble des capacités d'expertise en 
France, dont celle de l'IPSN et la nouvelle Direction générale de la sûreté nucléaire et de la radioprotection (DGSNR) qui devient, en 2006, l'Autorité de sûreté nucléaire (ASN).

6 L'intérêt majeur de l'ouvrage de Cyrille Foasso est d'offrir une vision d'ensemble des transformations de la "sûreté nucléaire » sur une période de soixante-dix ans. Étudier des évolutions sur une telle période, dans ce domaine, n'est pas sans risques ni difficultés. La première difficulté est celle de ne pas juste survoler une histoire internationale complexe, technique, institutionnelle et politique à la fois. L'auteur arrive à surmonter cette difficulté en s'intéressant en particulier au cas français, sans jamais s'enfermer dans le seul contexte national. La seconde des difficultés réside dans les sources sur lesquelles s'appuie l'histoire proposée dans l'ouvrage. Les difficultés de disposer de sources d'archives diversifiées laissent l'historien face à des discours, voire une histoire élaborée par le monde nucléaire lui-même. C'est là un problème que rencontrent tous les historiens travaillant sur le secteur nucléaire. L'ouvrage n'échappe pas totalement à cet écueil, l'auteur n'ayant pas eu accès à des archives permettant de rendre finement compte des débats contradictoires et des enjeux qui sont à l'origine de plusieurs décisions et dispositions dans le monde de la sûreté. Enfin, la troisième difficulté est elle aussi commune à l'ensemble des chercheurs en sciences humaines et sociales travaillant sur le nucléaire: trouver un positionnement qui permette d'échapper à l'injonction souvent faite aux chercheurs de se prononcer pour ou contre le nucléaire. L'auteur tient une position symétrique, cherchant à restituer les logiques propres au secteur sans rien occulter des difficultés ou des problèmes. L'ouvrage réussit ainsi à offrir un travail très intéressant sur le monde des experts de la sûreté et leur mode de représentation et de légitimation. Il aurait été toutefois intéressant de prendre en compte des transformations plus générales des modes d'expertise et de régulation à certains moments, notamment avec l'essor d'une forme institutionnelle particulière dans le monde de la régulation, l'« agence » et le développement d'une contre-expertise plurielle défendue par des associations. Ceci concerne par exemple le rôle de la critique des années 1970 dans la création de l'IPSN ou encore les transformations de l'organisation de l'expertise dans l'ensemble des secteurs sanitaires, techniques et environnementaux au moment de la création de l'IRSN et l'ASN. Enfin, l'ouvrage rend surtout compte des conceptions des experts et des transformations de la doctrine de la sûreté. La question des mises en œuvre de ces concepts et donc l'analyse des pratiques effectives (par exemple comment se déroule un "dialogue technique» entre concepteurs, producteurs et régulateurs) n'est pas traitée. Ceci est à l'évidence extrêmement difficile à traiter sans des fonds d'archives très fournis pour la partie historique ou sans un accès direct et libre au terrain pour la période plus contemporaine. Cela pose le problème plus général d'accès direct de ces chercheurs aux terrains industriels, dans des secteurs stratégiques comme celui du nucléaire étudié ici, mais aussi pour d'autres industries comme celle du pétrole ou du gaz, par exemple. Ces faiblesses de l'ouvrage n'enlèvent rien à son grand intérêt et à son apport indéniable. Il a le mérite d'avoir investi une question difficile par sa technicité et la multiplicité de ses enjeux et de pointer l'importance de disposer de travaux soucieux de comprendre les techniques à risques et les logiques qui président à leur développement. Cette publication représente désormais un travail indispensable pour tous ceux qui s'intéressent au nucléaire et, au-delà, aux questions des risques technoscientifiques et de leurs modes de régulation.

7 L'ouvrage de Cyrille Foasso pose finalement une question plus générale qui déborde le cas du nucléaire: comment étudier en SHS les grands secteurs industriels où les 
questions de risques structurent les activités et les modes d'organisation. L'ouvrage propose une réponse possible, rendre compte des logiques et des conceptions de gestion des risques des concepteurs, organisateurs et experts.

Un autre ouvrage, publié par Pierre Fournier, traitant également du nucléaire, propose une perspective radicalement différente: s'intéresser au travail ordinaire dans le secteur nucléaire. Ce travail est également issu d'une thèse de doctorat, en sociologie, soutenue en 1996. L'ambition de ce travail n'est pas de s'intéresser à l'ensemble du secteur nucléaire et aux modalités d'organisation du travail en général, mais de proposer une ethnographie d'une centrale nucléaire, celle de Marcoule. L'auteur positionne son travail en réponse à ce qu'il considère comme un paradoxe. D'une part, le nucléaire fait l'objet d'un débat politique et social récurrent, régulièrement relancé par des accidents majeurs (Tchernobyl, Fukushima). D'autre part, l'activité quotidienne des travailleurs du nucléaire reste peu connue en dehors de quelques travaux qui dénoncent la sous-traitance et l'intérim pour effectuer les tâches les plus dangereuses, débouchant sur une régulation collective des doses qui pèsent sur les plus fragiles. Pour combler cette lacune des travaux en SHS, l'auteur nous propose un travail riche et dense, fondé sur une observation participante réalisée, au début des années 1990, tour à tour en tant qu'intérimaire, stagiaire et ouvrier de la sous-traitance.

Pierre Fournier cherche tout d'abord à donner une description sensible et précise du travail dans le nucléaire à partir en particulier des opérations de "plongées ", à savoir l'intervention dans les zones contrôlées, présentant un risque de contamination ou d'irradiation radioactives. Il décrit alors un travail ouvrier qui peut paraître assez banal par les tâches à effectuer - réparation et entretien des installations, par exemple ; mais, en même temps, il s'agit d'un travail contraint par un ensemble de règles de sécurité qu'il n'est pas toujours aisé de s'approprier et de mettre en œuvre. À partir d'une série d'opérations, l'ouvrage décrit le travail de préparation et de coordination primordial avant chaque plongée, en rendant compte des solidarités collectives qui le structurent y compris dans l'habillage et le déshabillage -, ainsi que des différents positionnements et attitudes des agents en fonction des trajectoires individuelles et professionnelles et des effets générationnels. En ce qui concerne la mise en œuvre des règles de protection, l'ouvrage décrit les écarts entre la prescription et les pratiques effectives et les ajustements permanents qu'opèrent les travailleurs face aux différentes contraintes $\mathrm{du}$ réel. Pour comprendre plus finement la gestion des risques dans une centrale nucléaire, l'ouvrage s'intéresse ensuite à une catégorie bien particulière des travailleurs du nucléaire : ceux en charge d'édicter et de faire appliquer les règles de sécurité et de protection. Pour cela, il propose une socio-histoire des métiers de la radioprotection. L'un des résultats importants qu'il met en avant est l'évolution notable du profil des praticiens de la radioprotection. Alors que les premiers techniciens en charge de la radioprotection étaient issus du terrain et de la formation continue, les embauchés les plus récents $d u$ service sont généralement issus d'une formation initiale de radioprotection créée à la fin des années 1980. C'est un éloignement du terrain qui est décrit ici, avec des ingénieurs et des médecins du travail qui se tiennent dans la mesure du possible à distance des situations de risques, préférant les suivre de loin, en laissant ainsi une importante latitude aux travailleurs. Ils leur font ainsi porter la responsabilité de leur propre protection, favorisant un développement de pratiques d'auto-contrôle des opérateurs et une gestion entre soi des risques encourus. L'auteur montre que la sécurité n'est pas qu'une affaire de règles et de prescriptions, mais aussi une question 
de travail d'équipe et de solidarité. Ceci se manifeste notamment par un encadrement mutuel entre travailleurs et un apprentissage en pratiques sur le terrain.

À plusieurs reprises, Pierre Fournier affirme sa volonté de ne pas prendre parti pour ou contre le nucléaire, tenant ainsi une position de symétrie revendiquée par de nombreux travaux en sciences sociales. Dans son travail, il souhaite se démarquer ou nuancer les travaux sur les ouvriers du nucléaire menés, notamment, par Annie Thébaud-Mony. Cette dernière a montré, par une série d'enquêtes, comment le travail le plus dangereux dans le nucléaire pèse sur les sous-traitants, chargés des "plus sales boulots » et exposés à des doses de radioactivité importantes. Les observations de Fournier ne contredisent pas ce constat dans la mesure où il note également que ce sont les sous-traitants, parfois qualifiés et relativement bien payés, qui effectuent le plus gros des travaux en zones contrôlées. Toutefois, il insiste sur l'autonomie et les capacités d'ajustement de ces travailleurs qu'il refuse de décrire comme des travailleurs dominés, subissant une organisation du travail implacable. Cette approche, microsociologique, riche par les descriptions qu'elle fournit, tend de fait à mettre au second plan ce qu'une approche à un niveau plus macro peut montrer, à savoir la répartition des doses et des risques en fonction des statuts professionnels, le recours de plus en plus important à la sous-traitance et une intensification du travail induisant des risques supplémentaires, dénoncée par plusieurs acteurs. Si l'intérêt d'une étude fine dans ses descriptions est indéniable, elle permet difficilement de pointer ce que l'observateur ne voit pas lui-même. Elle permet encore moins d'interroger des évolutions de plus long terme, comme le développement de la sous-traitance et le vieillissement du parc électronucléaire qui entraîne une augmentation des activités de maintenance et donc de travail en zones à risques.

11 Les ouvrages de Cyrille Foasso et de Pierre Fournier, riches et stimulants, donnent à voir deux manières d'étudier les risques nucléaires, chacune étant non seulement légitime, mais indispensable pour saisir une partie de ce monde complexe et, au-delà, le fonctionnement d'une industrie de haute technologie présentant des risques avérés. Ils pointent au moins deux choses que l'on retrouve dans différentes industries à risque. La première est l'écart entre la cohérence et l'importance des règles de protection et de sûreté édictées par le monde de l'expertise et la régulation et la mise en œuvre de ces règles en pratiques, dans des conditions réelles de travail. Cet écart peut trouver différentes raisons, se distribuant sur un éventail allant de la difficulté à mettre en œuvre des règles non réalistes au quotidien au mépris pur et simple de l'employeur pour leur mise en œuvre. La seconde conclusion qui peut être tirée du cas du nucléaire est la variété des risques et leur prise en charge différenciée. Dit autrement, il n'y a pas une sûreté nucléaire mais des sûretés. La grande attention portée à la sécurité des réacteurs, étudiée par Cyrille Foasso, correspond à un mode de gestion du risque technique dans lequel la protection de la santé n'est pas l'objet principal. Cet important travail des experts vise avant tout à se prémunir contre des incidents techniques ou un accident grave pouvant coûter cher à l'industriel. Il s'agit ainsi de protéger avant tout les investissements réalisés et de construire une réputation de fiabilité technique indispensable à la construction des marchés dans ce type de secteur. La protection (radioprotection) des travailleurs et, notamment, les plus précaires d'entre eux, a souvent été le parent pauvre des programmes de recherche relatifs au risque. Même si l'expertise en radioprotection est organisée à l'échelle internationale depuis les années 1950, ses principaux acteurs ont souvent moins de poids dans les institutions que leurs homologues de la sûreté. Les deux ouvrages lus ensemble offrent une 
illustration saisissante de cette hiérarchie. Ils invitent à poursuivre les travaux sur le secteur nucléaire, en continuant à varier la focale d'étude pour saisir comment nos sociétés développent des risques industriels majeurs et comment, par le travail de différents acteurs, elles se retrouvent à faire avec ces risques pour le meilleur et pour le pire.

\section{AUTEURS}

SORAYA BOUDIA

Université de Paris Descartes - CERTFES3 